\title{
A novel direction-finding method for cyclostationary signals ${ }^{\text {in }}$
}

\author{
Ju-Hong Lee*, Yung-Ting Lee \\ Department of Electrical Engineering, National Taiwan University, Room 517, Building 2, Taipei 106, Taiwan, ROC
}

Received 3 March 2000; received in revised form 27 October 2000

\begin{abstract}
We develop a method based on the phase-SCORE algorithm of Agee et al. (Proc. IEEE 78 (April 1990) 753) to achieve direction finding for cyclostationary signals. Using the weight obtained from the phase-SCORE algorithm, we construct a subspace orthogonal to the subspace spanned by the direction vectors of uncorrelated signals of interest (SOIs). Finding the directions of the uncorrelated SOIs is then performed according to a subspace-fitting concept. For coping with the coherence between SOIs, we incorporate the proposed method with the well-known spatial smoothing algorithm of Shan et al. (IEEE Trans. Acoust. Speech Signal Process ASSP-33 (August 1985) 806) to alleviate the performance degradation due to coherence. Simulation examples for illustration and comparison are also provided. (C) 2001 Elsevier Science B.V. All rights reserved.
\end{abstract}

Keywords: Direction-finding; Cyclostationary signals; Subspace-fitting

\section{Introduction}

For finding signal directions using sensor arrays, many high-resolution methods have been developed based on eigenspace concepts $[5,6,9]$. Notable among them is the MUSIC algorithm [9]. Using the orthogonality between the signal subspace and the noise subspace, MUSIC can provide asymptotically unbiased estimates of the directions of arrival (DOAs) of incoherent and partially correlated sources.

Cyclostationarity [3], which is a statistical property possessed by most man-made communication signals, corresponds to the underlying periodicity

\footnotetext{
This work was supported by the National Science Council under Grant NSC89-2213-E002-084.

* Corresponding author.
}

arising from carrier frequencies and baud rates. Recently, signal cyclostationarity has been widely considered for direction finding, resulting in many techniques that inherently exhibit a signal-selective property. Notable among them is cyclic MUSIC (C-MUSIC) $[2,8]$ which uses a subspace-fitting concept to accommodate multiple signals having the same cycle frequency. In [4], it is shown that the beamforming techniques can also be used to estimate the DOAs of the SOIs. However, they always fail to separate the DOAs of spatially close SOIs.

In this paper, based on the phase-SCORE algorithm of [1] which was originally developed for performing adaptive blind array beamforming, we present the phase-SCORE-based method to find the directions of uncorrelated SOIs. Utilizing the weight of the phase-SCORE algorithm, we construct projection matrices whose columns are 
orthogonal to the subspace spanned by the direction vectors of the SOIs. Accordingly, the DOAs of the SOIs are effectively estimated even in the presence of strong SNOIs. In the situation with coherent signals, the original phase-SCORE algorithm may pass some linear combination of the coherent SOI components. This leads to the SOI being extracted with significant distortion. We use the spatial smoothing (SS) scheme, which does not depend on time stationarity of signals, to effectively alleviate the effect of the coherence between SOIs. Simulation results show the effectiveness of the proposed method.

\section{Background}

\subsection{Signal cyclostationarity}

The cyclic autocorrelation function of a signal $s(t)$ is defined by the infinite-time average

$$
r_{s s}(\alpha, \tau)=\left\langle s\left(t+\frac{\tau}{2}\right) s^{*}\left(t-\frac{\tau}{2}\right) \mathrm{e}^{-\mathbf{j} 2 \pi \alpha t}\right\rangle_{\infty},
$$

where $\langle\cdot\rangle_{\infty}=\lim _{T \rightarrow \infty} 1 / 2 T \int_{t=-T}^{T}(\cdot) \mathrm{d} t$, the superscript "*" denotes the complex conjugate. $s(t)$ is said to be cyclostationary if $r_{s s}(\alpha, \tau)$ does not equal zero at some time delay $\tau$ which can be any real number [3] and cycle frequency $\alpha \neq 0$. Many modulated signals exhibit cyclostationarity with cycle frequency equal to the twice the carrier frequency or multiples of the baud rate or combinations of these. When $r_{s s}(\alpha, \tau) \neq 0$ for $\alpha \neq 0$, we also say that $s(t)$ possesses the self-coherent property. Next, let $\boldsymbol{x}(t)$ denote the data vector received by a sensor array. Then, the corresponding cyclic autocorrelation matrix is given by

$\boldsymbol{R}_{x x}(\alpha, \tau)=\left\langle\boldsymbol{x}\left(t+\frac{\tau}{2}\right) \boldsymbol{x}^{\mathrm{H}}\left(t-\frac{\tau}{2}\right) \mathrm{e}^{-\mathrm{j} 2 \pi \alpha t}\right\rangle_{\infty}$,

where the superscript $\mathrm{H}$ denotes the conjugate transpose.

\subsection{Conventional cyclic MUSIC method}

Consider an $M$-element uniform linear array (ULA) excited by $d$ SOIs and $J$ SNOIs impinging on the array from $\left\{\theta_{1}, \theta_{2}, \ldots, \theta_{d}\right\}$ and $\left\{\phi_{1}\right.$, $\left.\phi_{2}, \ldots, \phi_{J}\right\}$ off broadside, respectively. The received array data can be written in vector form as

$\boldsymbol{x}(t)=\sum_{k=1}^{d} s_{k}(t) \boldsymbol{a}\left(\theta_{k}\right)+\sum_{l=1}^{J} q_{l}(t) \boldsymbol{a}\left(\phi_{l}\right)+\boldsymbol{n}(t)$,

where $s_{k}(t)$ and $q_{l}(t)$ denote the waveforms of the $k$ th SOI and the lth SNOI, $\boldsymbol{a}\left(\theta_{k}\right)$ and $\boldsymbol{a}\left(\phi_{l}\right)$ the corresponding direction vectors, respectively, and $\boldsymbol{n}(t)$ the noise vector. The autocorrelation matrix $\boldsymbol{R}_{x x}$ of $\boldsymbol{x}(t)$ is given by $\boldsymbol{R}_{x x}=E\left\{\boldsymbol{x}(t) \boldsymbol{x}^{\mathrm{H}}(t)\right\}$ which is of full rank with rank equal to $(d+J)$ when the $(d+J)$ signals are uncorrelated, and the sample version of $\boldsymbol{R}_{x x}$ computed by using $N$ data snapshots is given by $\hat{\boldsymbol{R}}_{x x, N}=1 / N \sum_{i=1}^{N} \boldsymbol{x}(i) \boldsymbol{x}^{\mathrm{H}}(i)$, where $\boldsymbol{x}(i) \mathrm{de}$ notes the data vector $\boldsymbol{x}(t)$ sampled at the time instant $i T_{s}$ with sampling interval $T_{s}$. Assume that the $d$ SOIs have a cycle frequency equal to $\alpha$, while the $J$ SNOIs and noise do not possess the cyclostationarity at $\alpha$ and are uncorrelated with the SOIs. Moreover, let the $d$ SOIs be far-field (in the Fraunhofer zone) and narrowband (total frequency extent is much smaller than the center frequency), the direction vector associated with the $k$ th SOI is given by

$\boldsymbol{a}\left(\theta_{k}\right)=\left[1, \mathrm{e}^{\mathrm{j} \Theta_{k}}, \mathrm{e}^{\mathrm{j} 2 \Theta_{k}}, \ldots, \mathrm{e}^{\mathrm{j}(M-1) \Theta_{k}}\right]^{\mathrm{T}}$,

where $\Theta_{k}=2 \pi d_{s} \sin \left(\theta_{k}\right) / \lambda_{k}$ denotes the relative phase shift between two adjacent array sensors due to the $k$ th SOI, $d_{s}$ the interelement spacing, and $\lambda_{k}$ the corresponding signal wavelength.

Let $\hat{\boldsymbol{R}}_{x x, N}(\alpha, \hat{\tau})$ represent the sample version of $\boldsymbol{R}_{x x}(\alpha, \tau)$ computed by using $N$ data snapshots, where $\hat{\tau}$ is used instead of $\tau$ for denoting the corresponding time delay in a sampled system. Then, the C-MUSIC method is implemented as follows: [3, pp. 214-215]. First, compute $\hat{\boldsymbol{R}}_{x x, N}(\alpha, \hat{\tau})=1$ / $N \sum_{i=1}^{N} \boldsymbol{x}(i) \boldsymbol{x}^{\mathrm{H}}(i-\hat{\tau}) \mathrm{e}^{-\mathrm{j} 2 \pi \alpha i T_{s}}$. Then, we perform the singular value decomposition (SVD) on $\hat{\boldsymbol{R}}_{x x, N}(\alpha, \hat{\tau})$ to obtain

$$
\hat{\boldsymbol{R}}_{x x, N}(\alpha, \hat{\tau})=\left[\begin{array}{ll}
\hat{\boldsymbol{S}}_{N} & \hat{\boldsymbol{G}}_{N}
\end{array}\right]\left[\begin{array}{cc}
\hat{\boldsymbol{\Sigma}}_{S, N} & \mathbf{0} \\
\mathbf{0} & \hat{\boldsymbol{\Sigma}}_{G, N}
\end{array}\right]\left[\begin{array}{ll}
\hat{\boldsymbol{V}}_{S, N} & \hat{\boldsymbol{V}}_{G, N}
\end{array}\right]^{\mathrm{H}},
$$


where $\left[\hat{\boldsymbol{S}}_{N} \hat{\boldsymbol{G}}_{\boldsymbol{N}}\right]$ and $\left[\hat{\boldsymbol{V}}_{S, N} \hat{\boldsymbol{V}}_{\boldsymbol{G}, N}\right]$ are two unitary matrices with the dimensions of $\hat{\boldsymbol{S}}_{N}$ and $\hat{\boldsymbol{V}}_{S, N}$ given by $M \times d$, and the dimensions of $\hat{\boldsymbol{G}}_{N}$ and $\hat{\boldsymbol{V}}_{G, N}$ given by $M \times(M-d)$. The diagonal elements of the diagonal matrices $\hat{\Sigma}_{S, N}$ and $\hat{\Sigma}_{G, N}$ are nonnegative and appear in the descending order. Finally, it is well known that finding the angles of the $d$ SOIs is equivalent to finding the $d$ minima of $\left\|\hat{\boldsymbol{G}}_{N}^{\mathrm{H}} \boldsymbol{a}(\psi)\right\|^{2}$ or the $d$ maxima of $\left\|\hat{\boldsymbol{S}}_{N}^{\mathrm{H}} \boldsymbol{a}(\psi)\right\|^{2}$, whichever is easier [7], where $\boldsymbol{a}(\psi)$ is the direction vector corresponding to an impinging angle $\psi$ and $\|\cdot\|$ denotes the vector norm operator. The obtained $d$ values of $\psi$ are then taken as the DOA estimates of the $d$ SOIs.

\subsection{The spatial smoothing scheme}

Let an $M$-element ULA be divided into overlapping subarrays of size $M_{s}$, with sensor elements $\left\{k, \ldots, k+M_{s}-1\right\}$ forming the $k$ th subarray. Let $\boldsymbol{x}_{k}(t)$ be the data vector received by the $k$ th subarray and $\boldsymbol{R}_{x x}(k)$ be the autocorrelation matrix of $\boldsymbol{x}_{k}(t)$. When the SS scheme of [10] is performed on $\hat{\boldsymbol{R}}_{x x, N}$, we obtain the spatial smoothed matrix by taking $\overline{\boldsymbol{R}}_{x x, N}=1 / L \sum_{k=1}^{L} \hat{\boldsymbol{R}}_{x x, N}(k)$, where $L=M-M_{s}+1$ and $\hat{\boldsymbol{R}}_{x x, N}(k)$ denotes the sample version of $\boldsymbol{R}_{x x}(k)$ computed by using $N$ data snapshots. In order to successfully decorrelate $K$ coherent SOIs, the conditions $L \geqslant K$ and $M_{s} \geqslant(d+J+1)$ must be held. Then, $\overline{\boldsymbol{R}}_{x x, N}$ is used instead of $\hat{\boldsymbol{R}}_{x x, N}$ for performing direction finding.

\section{The proposed method}

According to the phase-SCORE algorithm [1], the weight required for retrieving one $\mathrm{SOI}$ is given by the dominant eigenvector of

$\hat{\boldsymbol{R}}_{x x, N}^{-1} \hat{\boldsymbol{R}}_{x u, N}$,

where $\hat{\boldsymbol{R}}_{x u, N}=1 / N \sum_{i=1}^{N} \boldsymbol{x}(i) \boldsymbol{u}^{\mathrm{H}}(i)$ represents the sample cross-correlation matrix of $\boldsymbol{x}(i)$ and $\boldsymbol{u}(i)=\boldsymbol{x}(i-\hat{\tau}) \mathrm{e}^{\mathrm{j} 2 \pi \alpha i T_{s}}$. Accordingly, in the case of retrieving $d$ SOIs, the required weights are given by the $d$ dominant eigenvectors of (6). Under the assumption of ergodicity, we have from (1) to (3) that $\boldsymbol{R}_{x u} \equiv \lim _{N \rightarrow \infty} \hat{\boldsymbol{R}}_{x u, N}=\lim _{N \rightarrow \infty} \frac{1}{N} \sum_{i=1}^{N} \boldsymbol{x}(i) \boldsymbol{x}^{\mathrm{H}}(i-\hat{\tau}) \mathrm{e}^{-\mathrm{j} 2 \pi \alpha i T_{s}}$

$$
\begin{aligned}
= & \sum_{k=1}^{d} \sum_{h=1}^{d}\left\{\lim _{N \rightarrow \infty} \frac{1}{N} \sum_{i=1}^{N} s_{k}(i) s_{h}^{*}(i-\hat{\tau}) \mathrm{e}^{-\mathrm{j} 2 \pi \alpha i T_{s}}\right\} \\
& \times \boldsymbol{a}\left(\theta_{k}\right) \boldsymbol{a}^{\mathrm{H}}\left(\theta_{h}\right) \\
= & \sum_{k=1}^{d} \sum_{h=1}^{d} r_{s_{k} s_{h}}(\alpha, \tau) \mathrm{e}^{-\mathrm{j} \pi \alpha \tau} \boldsymbol{a}\left(\theta_{k}\right) \boldsymbol{a}^{\mathrm{H}}\left(\theta_{h}\right),
\end{aligned}
$$

where $r_{s_{k} s_{h}}(\alpha, \tau)$ is the cyclic correlation function of $s_{k}(t)$ and $s_{h}(t)$. The terms including the SNOIs and noise disappear as $N \rightarrow \infty$ from (7) since they are not cyclostationary at $\alpha$ and are uncorrelated with the SOIs. From (6) and (7), we obtain

$$
\boldsymbol{R}_{x x}^{-1} \boldsymbol{R}_{x u}=\sum_{k=1}^{d} \sum_{h=1}^{d} r_{s_{k} s_{h}}(\alpha, \tau) \mathrm{e}^{-\mathbf{j} \pi \alpha \tau}\left[\boldsymbol{R}_{x x}^{-1} \boldsymbol{a}\left(\theta_{k}\right)\right] \boldsymbol{a}^{\mathrm{H}}\left(\theta_{h}\right)
$$

when $N$ approaches infinity, where $\boldsymbol{R}_{x x} \equiv$ $\lim _{N \rightarrow \infty} \hat{\boldsymbol{R}}_{x x, N}$. It is obvious from (8) that $\boldsymbol{R}_{x x}^{-1} \boldsymbol{R}_{x u}$ is a matrix with rank $d$ since $\boldsymbol{R}_{x x}$ is of full rank because the signals are uncorrelated. Moreover, the corresponding $d$ dominant eigenvectors form a basis of the SOI signal subspace spanned by $\left\{\boldsymbol{R}_{x x}^{-1} \boldsymbol{a}\left(\theta_{k}\right), k=1,2, \ldots, d\right\}$. Let $\hat{\boldsymbol{e}}_{k, N}$ represent the $k$ th dominant eigenvector of $\hat{\boldsymbol{R}}_{x x, N}^{-1} \hat{\boldsymbol{R}}_{x u, N}$. We then construct a projection matrix which projects a vector onto the subspace spanned by the SNOIs and the noise as follows:

$\hat{\boldsymbol{P}}_{N}=\boldsymbol{I}-\hat{\boldsymbol{A}}_{N}\left\{\hat{\boldsymbol{A}}_{N}^{\mathrm{H}} \hat{\boldsymbol{A}}_{N}\right\}^{-1} \hat{\boldsymbol{A}}_{N}^{\mathrm{H}}$,

where $\hat{\boldsymbol{A}}_{N}=\left[\hat{\boldsymbol{R}}_{x x, N} \hat{\boldsymbol{e}}_{1, N}, \ldots, \hat{\boldsymbol{R}}_{x x, N} \hat{\boldsymbol{e}}_{d, N}\right]$ and $\boldsymbol{I}$ is the identity matrix with appropriate size. Using the subspace-fitting concept of [9], the $d$ estimates of directions of the SOIs are then obtained by taking the $d$ maxima of

$F=\frac{1}{\boldsymbol{a}^{\mathrm{H}}(\psi) \hat{\boldsymbol{P}}_{N} \boldsymbol{a}(\psi)}$

with respect to $\psi$, where $\boldsymbol{a}(\psi)$ represents the direction vector of angle $\psi$ off array broadside.

Next, consider the situation where the SOIs are coherent. The coherence between SOIs leads to the direction vectors of coherent SOIs, even from different impinging angles, being linearly combined and, hence, results in ambiguity for direction finding. To alleviate the performance degradation due to coherence, we resort to the SS scheme described 
in Section 2.3. The proposed method is further combined with the SS scheme. First, we perform the SS scheme on $\hat{\boldsymbol{R}}_{x x, N}$ and $\hat{\boldsymbol{R}}_{x u, N}$ to obtain $\overline{\boldsymbol{R}}_{x x, N}$ and $\overline{\boldsymbol{R}}_{x u, N}$, respectively. Then, $\overline{\boldsymbol{R}}_{x x, N}^{-1} \overline{\boldsymbol{R}}_{x u, N}$ instead of $\hat{\boldsymbol{R}}_{x x, N}^{-1} \hat{\boldsymbol{R}}_{x u, N}$ is used by the proposed method for carrying out direction finding.

\section{Discussion on the proposed method}

Consider the performance of the proposed method. For simplicity, we assume that only one SOI and one SNOI exist, i.e., $d=1$ and $J=1$ for (3). Thus, the $\boldsymbol{x}(t)$ of (3) becomes

$\boldsymbol{x}(t)=s(t) \boldsymbol{a}(\theta)+q(t) \boldsymbol{a}(\phi)+\boldsymbol{n}(t)$.

Then, we have the sample cyclic autocorrelation matrix as

$$
\begin{gathered}
\hat{\boldsymbol{R}}_{x x, N}(\alpha, \hat{\tau}) \\
=\frac{1}{N} \sum_{i=1}^{N}\{[s(i) \boldsymbol{a}(\theta)+q(i) \boldsymbol{a}(\phi)+\boldsymbol{n}(i)] \\
\times[\{s(i-\hat{\tau}) \boldsymbol{a}(\theta)+q(i-\hat{\tau}) \boldsymbol{a}(\phi) \\
+\boldsymbol{n}(i-\hat{\tau})\}]^{\mathrm{H}} \mathrm{e}^{\left.-\mathbf{j} 2 \pi \alpha i T_{s}\right\}} \\
=\hat{r}_{s s, N}(\alpha, \hat{\tau}) \boldsymbol{a}(\theta) \boldsymbol{a}^{\mathrm{H}}(\theta)+\hat{r}_{q q, N}(\alpha, \hat{\tau}) \boldsymbol{a}(\phi) \boldsymbol{a}^{\mathrm{H}}(\phi) \\
+\hat{\boldsymbol{R}}_{n n, N}(\alpha, \hat{\tau})+\hat{\boldsymbol{C}}_{1, N},
\end{gathered}
$$

where $\hat{\boldsymbol{C}}_{1, N}$ contains all the cross-terms. Substituting (12) into (6) yields

$$
\begin{aligned}
\hat{\boldsymbol{R}}_{x x, N}^{-1} \hat{\boldsymbol{R}}_{x u, N}= & \hat{\boldsymbol{R}}_{x x, N}^{-1} \hat{\boldsymbol{R}}_{x x, N}(\alpha, \hat{\tau}) \\
= & \hat{r}_{s s, N}(\alpha, \hat{\tau})\left[\hat{\boldsymbol{R}}_{x x, N}^{-1} \boldsymbol{a}(\theta)\right] \boldsymbol{a}^{\mathrm{H}}(\theta) \\
& +\hat{r}_{q q, N}(\alpha, \hat{\tau})\left[\hat{\boldsymbol{R}}_{x x, N}^{-1} \boldsymbol{a}(\phi)\right] \boldsymbol{a}^{\mathrm{H}}(\phi) \\
& +\hat{\boldsymbol{R}}_{x x, N}^{-1} \hat{\boldsymbol{R}}_{n n, N}(\alpha, \hat{\tau})+\hat{\boldsymbol{C}}_{2, N},
\end{aligned}
$$

where $\hat{\boldsymbol{C}}_{2, N}=\hat{\boldsymbol{R}}_{x x, N}^{-1} \hat{\boldsymbol{C}}_{1, N} . \Theta$ and $\Phi$ denote the relative phase shifts between two adjacent array sensors due to the SOI and SNOI, respectively. We note that the dominant eigenvectors of (12) and (13) are proportional to $\boldsymbol{a}(\theta)$ and $\boldsymbol{R}_{x x}^{-1} \boldsymbol{a}(\theta)$, respectively, when $N$ approaches infinity. From (12) and (13), we have

$$
\begin{aligned}
\hat{\boldsymbol{R}}_{x x, N}(\alpha, \hat{\tau}) \boldsymbol{a}(\theta) \\
=\left\{\boldsymbol{a}^{\mathrm{H}}(\theta) \boldsymbol{a}(\theta)\right\}\left\{\hat{r}_{s s, N}(\alpha, \hat{\tau}) \boldsymbol{a}(\theta)\right. \\
\left.+\left(\frac{\boldsymbol{a}^{\mathrm{H}}(\phi) \boldsymbol{a}(\theta)}{\boldsymbol{a}^{\mathrm{H}}(\theta) \boldsymbol{a}(\theta)}\right) \hat{r}_{q q, N}(\alpha, \hat{\tau}) \boldsymbol{a}(\phi)\right\} \\
+\hat{\boldsymbol{R}}_{n n, N}(\alpha, \hat{\tau}) \boldsymbol{a}(\theta)+\hat{\boldsymbol{C}}_{1, N} \boldsymbol{a}(\theta)
\end{aligned}
$$

and

$$
\begin{aligned}
\hat{\boldsymbol{R}}_{x x, N}^{-1} & \hat{\boldsymbol{R}}_{x u, N}\left[\hat{\boldsymbol{R}}_{x x, N}^{-1} \boldsymbol{a}(\theta)\right] \\
= & \left\{\boldsymbol{a}^{\mathrm{H}}(\theta) \hat{\boldsymbol{R}}_{x x, N}^{-1} \boldsymbol{a}(\theta)\right\}\left\{\hat{r}_{s s, N}(\alpha, \hat{\tau})\left[\hat{\boldsymbol{R}}_{x x, N}^{-1} \boldsymbol{a}(\theta)\right]\right. \\
& \left.+\left(\frac{\boldsymbol{a}^{\mathrm{H}}(\phi) \hat{\boldsymbol{R}}_{x x, N}^{-1} \boldsymbol{a}(\theta)}{\boldsymbol{a}^{\mathrm{H}}(\theta) \hat{\boldsymbol{R}}_{x x, N}^{-1} \boldsymbol{a}(\theta)}\right) \hat{r}_{q q, N}(\alpha, \hat{\tau})\left[\hat{\boldsymbol{R}}_{x x, N}^{-1} \boldsymbol{a}(\phi)\right]\right\} \\
& +\hat{\boldsymbol{R}}_{x x, N}^{-1} \hat{\boldsymbol{R}}_{n n, N}(\alpha, \hat{\tau}) \hat{\boldsymbol{R}}_{x x, N}^{-1} \boldsymbol{a}(\theta)+\hat{\boldsymbol{C}}_{2, N} \hat{\boldsymbol{R}}_{x x, N}^{-1} \boldsymbol{a}(\theta),
\end{aligned}
$$

respectively. We note that (14) and (15) are the equations for showing the dominance of the SOI by using the C-MUSIC and the proposed method, respectively. Due to the fact that $\left|\boldsymbol{a}^{\mathrm{H}}(\phi) \hat{\boldsymbol{R}}_{x x, N}^{-1} \boldsymbol{a}(\theta) / \boldsymbol{a}^{\mathrm{H}}(\theta) \hat{\boldsymbol{R}}_{x x, N}^{-1} \boldsymbol{a}(\theta)\right| \ll \mid \boldsymbol{a}^{\mathrm{H}}(\phi) \boldsymbol{a}(\theta) / \boldsymbol{a}^{\mathrm{H}}(\theta) \boldsymbol{a}(\theta)$ | when the number of array sensors is large enough as shown in the appendix, we would expect from (14) and (15) that the proposed method reduces the effect of the SNOI much more than C-MUSIC. Eq. (A.6) in the appendix also reveals that the proposed method outperforms C-MUSIC, especially in the presence of strong SNOI when only finite data samples are available.

\section{Simulation examples}

In this section, several simulation examples are presented for illustration and comparison. Consider an 8-element ULA with interelement spacing equal to the half wavelength of the SOIs. $\tau=0$ is used for simplicity. The noise is white Gaussian with zero mean and unit variance. Each example it contains the simulations using $\boldsymbol{R}_{x x}$ and $\boldsymbol{R}_{x u}$, and the simulations obtained by averaging 50 independent runs using $\hat{\boldsymbol{R}}_{x x, N}$ and $\hat{\boldsymbol{R}}_{x u, N}$. 
Example 1. The sampling rate is set to 1. Two uncorrelated SOIs impinging on the array from $5^{\circ}$ and $25^{\circ}$ off broadside are BPSK signals having raised cosine spectrum with roll-off factor $=1$, baud rate $=0.2$, and signal-to-noise ratio (SNR) $=0 \mathrm{~dB}$. A SNOI impinging on the array from $-20^{\circ}$ off broadside is also a BPSK signal having raised cosine spectrum with roll-off factor $=1$, baud rate $=0.25$, and $\mathrm{SNR}=9 \mathrm{~dB}$. The cycle frequency is set to the baud rate of SOIs, that is, $\alpha=0.2$. Moreover, 3000 data samples are used to obtain the required sample correlation matrices. Fig. 1 plots the resulting bearing spectra using the
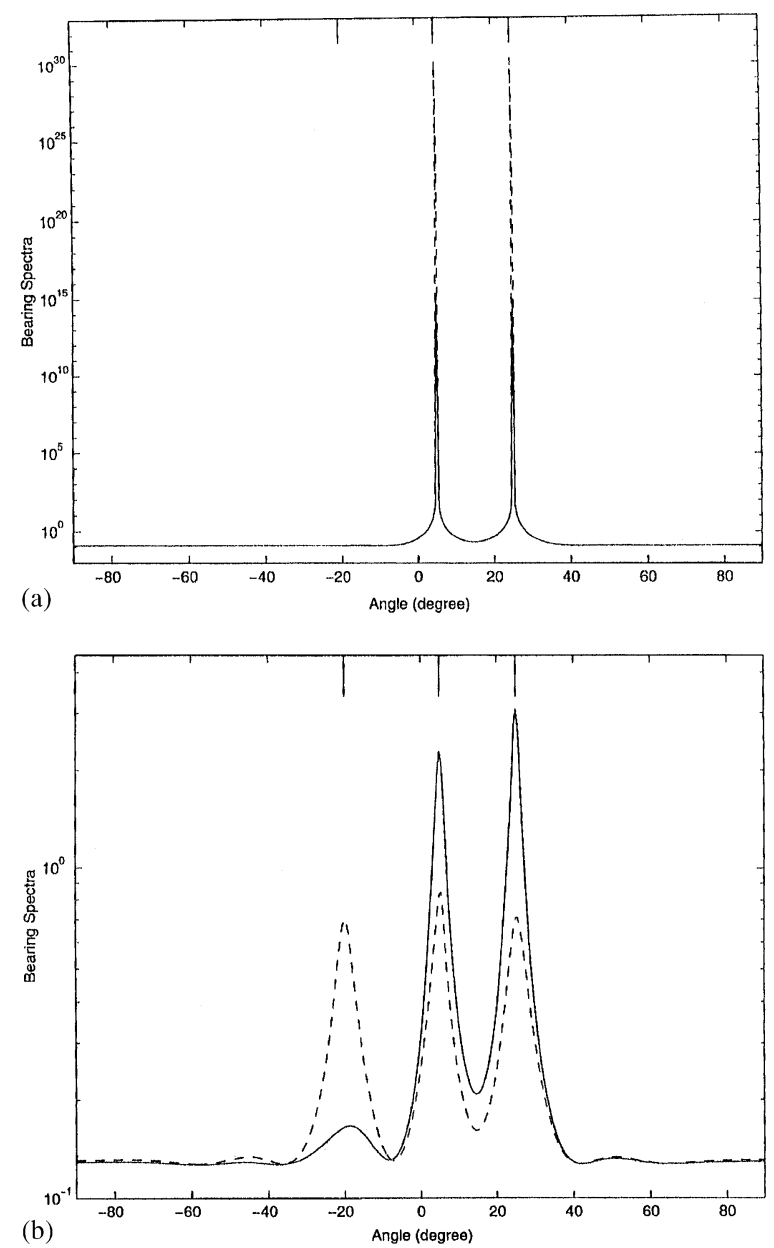

Fig. 1. Bearing spectra versus the impinging angle for Example 1. (a) Using ensemble correlation matrices, (b) using 3000 data samples. ( $\square$ ) the proposed method; (- - $)$ C-MUSIC. proposed method. The results of using C-MUSIC are also provided for comparison. We observe from Fig. 1 that the proposed method and C-MUSIC can provide accurate DOA estimation for the SOIs when ensemble correlation matrices are used. However, the former outperforms the latter in the presence of strong SNOI when only finite data samples are available.

Example 2. Here, we consider the same simulation scenario as that of Example 1 except that the two SOIs are coherent. The 8-element array are

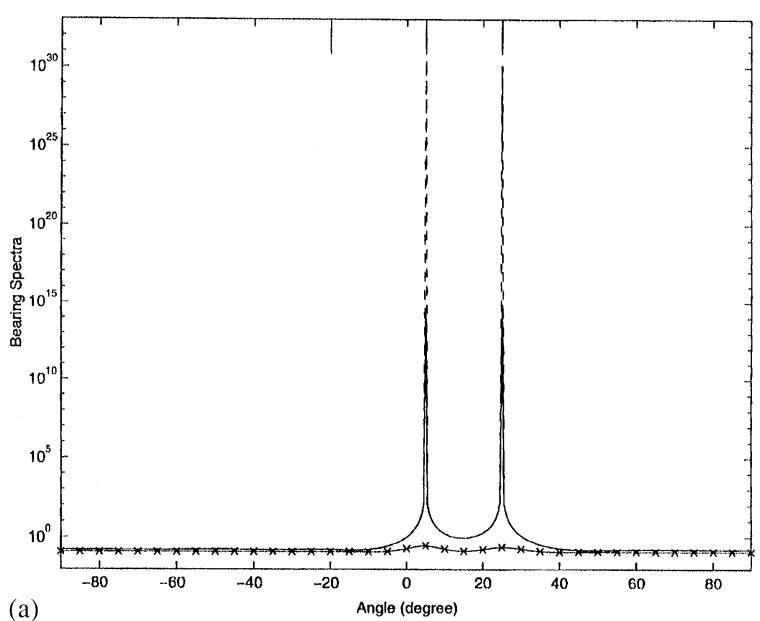

(a)

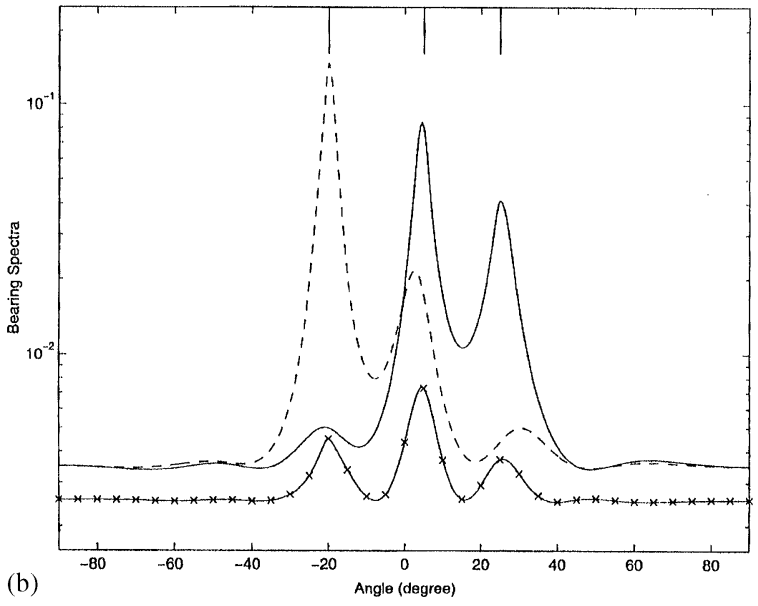

Fig. 2. Bearing spectra versus the impinging angle for Example 2. (a) Using ensemble correlation matrices, (b) using 3000 data samples. (- ${ }^{-}$the proposed method; $\left(--^{-}\right)$SS C-MUSIC; $(-x-)$ C-MUSIC. 
grouped into three subarrays of size 6 with sensor elements $\{i, i+1, \ldots, i+5\}$ forming the $i$ th subarray. Fig. 2 depicts the resulting bearing spectra using the proposed method in conjunction with the SS scheme. The results of using C-MUSIC and $\mathrm{C}$-MUSIC in conjunction with the SS scheme (SS C-MUSIC) are also given for comparison. From Fig. 2(a), we note that both of the proposed methods with the SS scheme and the SS C-MUSIC can successfully find the bearings of the coherent SOIs when ensemble correlation matrices are used. Nevertheless, as shown in Fig. 2(b), the former outperforms the latter when only finite data samples are available. The ambiguity of C-MUSIC in the presence of coherent SOIs can also be observed from Fig. 2.

\section{Appendix}

Using (11) and assuming $N$ large enough, we have

$\hat{\boldsymbol{R}}_{x x, N} \approx \sigma_{s}^{2} \boldsymbol{a}(\theta) \boldsymbol{a}^{\mathrm{H}}(\theta)+\hat{\boldsymbol{R}}_{j n, N}$,

where $\hat{\boldsymbol{R}}_{j n, N} \approx \sigma_{j}^{2} \boldsymbol{a}(\phi) \boldsymbol{a}^{\mathrm{H}}(\phi)+\sigma_{n}^{2} \boldsymbol{I}$ denotes the correlation matrix due to the SNOI and noise. $\sigma_{s}^{2}, \sigma_{j}^{2}$, and $\sigma_{n}^{2}$ are the SOI, SNOI, and noise powers, respectively. Taking the inverse of (A.1) and using the matrix inversion lemma yields

$\hat{\boldsymbol{R}}_{x x, N}^{-1} \approx \hat{\boldsymbol{R}}_{j n, N}^{-1}-\frac{\sigma_{s}^{2} \hat{\boldsymbol{R}}_{j n, N}^{-1} \boldsymbol{a}(\theta) \boldsymbol{a}^{\mathrm{H}}(\theta) \hat{\boldsymbol{R}}_{j n, N}^{-1}}{1+\sigma_{s}^{2} \boldsymbol{a}^{\mathrm{H}}(\theta) \hat{\boldsymbol{R}}_{j n, N}^{-1} \boldsymbol{a}(\theta)}$.

Multiplying both sides of (A.2) by $\boldsymbol{a}(\theta)$ and performing some algebraic manipulations yields

$\hat{\boldsymbol{R}}_{x x, N}^{-1} \boldsymbol{a}(\theta) \approx \frac{\hat{\boldsymbol{R}}_{j n, N}^{-1} \boldsymbol{a}(\theta)}{1+\sigma_{s}^{2} \boldsymbol{a}^{\mathrm{H}}(\theta) \hat{\boldsymbol{R}}_{j n, N}^{-1} \boldsymbol{a}(\theta)}$.

Similarly, we can obtain

$\hat{\boldsymbol{R}}_{j n, N}^{-1} \approx \frac{1}{\sigma_{n}^{2}}\left[\boldsymbol{I}-\frac{\sigma_{j}^{2} \boldsymbol{a}(\phi) \boldsymbol{a}^{\mathrm{H}}(\phi)}{\sigma_{n}^{2}+M \sigma_{j}^{2}}\right]$ since $\boldsymbol{a}^{\mathrm{H}}(\phi) \boldsymbol{a}(\phi)=M$ and, hence,

$\hat{\boldsymbol{R}}_{j n, N}^{-1} \boldsymbol{a}(\theta) \approx \frac{1}{\sigma_{n}^{2}}\left[\boldsymbol{a}(\theta)-\frac{\sigma_{j}^{2} \boldsymbol{a}^{\mathrm{H}}(\phi) \boldsymbol{a}(\theta)}{\sigma_{n}^{2}+M \sigma_{j}^{2}} \boldsymbol{a}(\phi)\right]$.

It follows from (A.3) and (A.5) that

$$
\begin{aligned}
& \left|\frac{\boldsymbol{a}^{\mathrm{H}}(\phi) \hat{\boldsymbol{R}}_{x x, N}^{-1} \boldsymbol{a}(\theta)}{\boldsymbol{a}^{\mathrm{H}}(\theta) \hat{\boldsymbol{R}}_{x x, N}^{-1} \boldsymbol{a}(\theta)}\right| \approx\left|\frac{\boldsymbol{a}^{\mathrm{H}}(\phi) \hat{\boldsymbol{R}}_{j n, N}^{-1} \boldsymbol{a}(\theta)}{\boldsymbol{a}^{\mathrm{H}}(\theta) \hat{\boldsymbol{R}}_{j n, N}^{-1} \boldsymbol{a}(\theta)}\right| \\
& \quad \approx\left|\frac{\sigma_{n}^{2} /\left(\sigma_{n}^{2}+M \sigma_{j}^{2}\right) \boldsymbol{a}^{\mathrm{H}}(\phi) \boldsymbol{a}(\theta)}{M-\sigma_{j}^{2} /\left(\sigma_{n}^{2}+M \sigma_{j}^{2}\right)\left|\boldsymbol{a}^{\mathrm{H}}(\phi) \boldsymbol{a}(\theta)\right|^{2}}\right| \\
& =\left|\frac{\sigma_{n}^{2} \boldsymbol{a}^{\mathrm{H}}(\phi) \boldsymbol{a}(\theta)}{M\left(\sigma_{n}^{2}+M \sigma_{j}^{2}\right)-\sigma_{j}^{2}\left|\boldsymbol{a}^{\mathrm{H}}(\phi) \boldsymbol{a}(\theta)\right|^{2}}\right| \\
& =\left|\frac{\boldsymbol{a}^{\mathrm{H}}(\phi) \boldsymbol{a}(\theta)}{\boldsymbol{a}^{\mathrm{H}}(\theta) \boldsymbol{a}(\theta)}\right| \times \frac{M}{M+\left(\sigma_{j}^{2} / \sigma_{n}^{2}\right)\left(M^{2}-\left|\boldsymbol{a}^{\mathrm{H}}(\phi) \boldsymbol{a}(\theta)\right|^{2}\right)} \\
& =\left|\frac{\boldsymbol{a}^{\mathrm{H}}(\phi) \boldsymbol{a}(\theta)}{\boldsymbol{a}^{\mathrm{H}}(\theta) \boldsymbol{a}(\theta)}\right| \times \frac{1}{1+M \sigma_{j}^{2} / \sigma_{n}^{2}\left(1-\left(\left|\boldsymbol{a}^{\mathrm{H}}(\phi) \boldsymbol{a}(\theta)\right|^{2} / M^{2}\right)\right)} .
\end{aligned}
$$

Since the term $1 / 1+M \sigma_{j}^{2} / \sigma_{n}^{2}\left(1-\left(\left|\boldsymbol{a}^{\mathrm{H}}(\phi) \boldsymbol{a}(\theta)\right|^{2} / M^{2}\right)\right)$ in (A.6) is much less than one when the number $M$ of array sensors is large enough or $\sigma_{n}^{2} \ll \sigma_{j}^{2}$, i.e., strong SNOI. As a result, we have that $\left|\boldsymbol{a}^{\mathrm{H}}(\phi) \hat{\boldsymbol{R}}_{x x, N}^{-1} \boldsymbol{a}(\theta) / \boldsymbol{a}^{\mathrm{H}}(\theta) \hat{\boldsymbol{R}}_{x x, N}^{-1} \boldsymbol{a}(\theta)\right| \ll\left|\boldsymbol{a}^{\mathrm{H}}(\phi) \boldsymbol{a}(\theta) / \boldsymbol{a}^{\mathrm{H}}(\theta) \boldsymbol{a}(\theta)\right|$.

\section{References}

[1] B.G. Agee, S.V. Schell, W.A. Gardner, Spectral self-coherence restoral: a new approach to blind adaptive signal extraction using antenna arrays, Proc. IEEE 78 (April 1990) 753-767.

[2] W.A. Gardner, Simplification of MUSIC and ESPRIT by exploitation of cyclostationarity, Proc. IEEE 76 (July 1988) 845-847.

[3] W.A. Gardner, Cyclostationarity in communications and signal processing, IEEE Press, New York, 1994.

[4] G. Gelli, L. Izzo, A cyclic beamforming method for signal DOA estimation, Proceedings of the Sixth European Signal Processing Conference, 1992, pp. 1805-1808.

[5] V.F. Pisarenko, The retrieval of harmonics from a covariance function, Geophys. J. Roy. Astron. Soc. 33 (1973) 247-266.

[6] R. Roy, T. Kailath, ESPRIT - estimation of signal parameter via rotation invariance techniques, IEEE Trans. Acoust. Speech Signal Process. 37 (July 1989) 984-995. 
[7] S.V. Schell, Exploitation of spectral correlation for signalselective direction finding, Ph.D. Thesis, Department of Electrical Engineering and Computer Science, University of California, Davis, CA, 1990.

[8] S.V. Schell, R.A. Calabretta, W.A. Gardner, B.G. Agee, Cyclic MUSIC algorithm for signal-selective direction estimation, Proceedings of the ICASSP, May 1989, pp. $2278-2281$.
[9] R.O. Schmidt, Multiple emitter location and signal parameter estimation, IEEE Trans. Antennas Propagation 34 (March 1986) 276-280.

[10] T.-J. Shan, W. Wax, T. Kailath, On spatial smoothing for direction-of-arrival estimation of coherent signals, IEEE Trans. Acoust. Speech Signal Process. ASSP-33 (August 1985) 806-811. 